\title{
EL GÉNERO ELAPHROTHRIPS BUFFA, 1909 (INSECTA, THYSANOPTERA, TUBULIFERA) EN AMÉRICA CENTRAL, DISCUSIÓN DEL CONCEPTO DE ESPECIE Y DESCRIPCIÓN DE ESPECIES NUEVAS PARA COSTA RICA
}

\author{
Axel P. RETANA-SALAZAR, ${ }^{1,3}$ Gerardo A. SOTO-RODRÍGUEZ ${ }^{1,2}$ y J. AleXander \\ RODRÍGUEZ-ARRIETA ${ }^{1,4,5}$
}

\author{
${ }^{1}$ Centro de Investigación en Estructuras Microscópicas (CIEMic), Ciudad de la Investigación, Universidad de \\ Costa Rica 2060. \\ ${ }^{2}$ Hypericum Pharma, S.A. San José, Costa Rica. Apartado 1237-2050 \\ ${ }^{3}$ Escuela de Nutrición, Facultad de Medicina, Ciudad de la Investigación, Universidad de Costa Rica 2060, San \\ Pedro de Montes de Oca, San José, Costa Rica \\ ${ }^{4}$ Escuela de Biología, Universidad de Costa Rica 2060, San Pedro de Montes de Oca, San José, Costa Rica. \\ ${ }^{5}$ Escuela de Ciencias Agrarias, Universidad Nacional. Heredia, Costa Rica. <axel.retana@ucr.ac.cr>
}

Recibido: 14/08/2014; aceptado: 25/02/2015

Retana-Salazar, A. P., Soto-Rodríguez, G. A. \& Rodriguez-Arrieta, J. A. 2015. El género Elaphrothrips Buffa, 1909 (Insecta, Thysanoptera, Tubulifera) en América Central, discusión del concepto de especie y descripción de especies nuevas para Costa Rica. Acta Zoológica Mexicana (n. s.), 31(2): 198-207.

RESUMEN. Se analiza el concepto de especie dentro del género Elaphrothrips, así como los problemas de criterios taxonómicos relacionados a este grupo. Además, se discuten las variaciones morfológicas del mismo y se presentan las características de los grupos de especies en el género. Se revisó el material de Elaphrothrips que ha sido recolectado y se describen tres especies nuevas procedentes de material recolectado en Costa Rica pero depositado en la colección del Senckenberg Museum en Alemania.

Palabras clave: Elaphrothrips, Idolothripinae, especies nuevas, grupos, Costa Rica.

\section{INTRODUCCIÓN}

El género Elaphrothrips es uno de los más grandes dentro del suborden Tubulifera. Frecuentemente confundido con las especies de Idolothrips (Mound 1968). La revisión más extensa del género la efectúa Johansen (1982) con especial énfasis en las especies de América. Mound \& Marullo (1996) consideran que el género en Costa Rica incluye tan solo siete especies, de las cuales grandis aparece como sinónimo de foveicollis en la página 221 y en las subsiguientes se considera como especie válida (Bhatti 1998). Retana-Salazar \& Soto-Rodríguez (2002) describen tres especies nuevas para el género y presentan una clave que incluye 16 especies para Costa Rica.

El trabajo de Retana-Salazar \& Soto-Rodríguez (2002) se fundamenta en una revisión de material y una extensa recolección en este país centroamericano. Sin embargo, no hay consenso en el criterio de especie utilizado en este género como lo señalan Mound \& Marullo (1996).
Retana-Salazar, A. P., Soto-Rodríguez, G. A. \& Rodriguez-Arrieta, J. A. 2015. The genus Elaphrothrips, Buffa 1909 (Insecta, Thysanoptera, Tubulifera) in Central America, discussion of the concept of species and description of new species for Costa Rica. Acta Zoológica Mexicana (n. s.), 31(2): 198-207.

ABSTRACT. In this paper, the species concept within genus Elaphrothrips is discussed, as well as problems of taxonomic criteria related to this group. In addition, the morphological variations and the characteristics of the species groups that occur in the genus, are also discussed. Furthermore, specimens of Elaphrothrips sampled in Costa Rica which belong to the collection of the Senckenberg Museum Frankfurt, Germany, were studied and three new species were found and described. Key words: Elaphrothrips, Idolothripinae, new species, groups, Costa Rica.

Los objetivos de este trabajo fueron analizar la definición de especie, las variaciones morfológicas y los grupos dentro del género Elaphrothrips, así como la descripción de tres especies nuevas halladas en el material de Costa Rica depositado en el Museo de Senckenberg, en Frankfurt, Alemania.

\section{MATERIAL Y MÉTODOS}

Para el presente trabajo se revisó el material de Elaphrothrips depositado en la Colección Institucional de Thysanoptera de la Universidad de Costa Rica (CITUCR, CIEMIC). El material utilizado para las descripciones de las especies nuevas se halla depositado en su totalidad en el Senckenberg Museum, Frankfurt (SMF), Alemania, el cual fue revisado entre los años 1996 a 2006, período en el que se encontraba depositado en calidad de préstamo en la Colección Institucional de Thysanoptera de la Universidad de Costa Rica. Este material recolectado por E. 
Klein en los años 70 está registrado en el SMF con los códigos T 6582 SMF, T 6594 SMF y T 6630 SMF.

\section{RESULTADOS}

\section{El grupo cesari como un linaje dentro de Elaphrothrips.}

Dos de las especies descritas por Retana-Salazar \& SotoRodríguez (2002) presentaron caracteres que fragmentan la poca consistencia de la diagnosis del género y deben considerarse en un grupo aparte de los considerados por Johansen (1986).

Las especies cesari y lorraineae presentan la característica de que los ojos no sobresalen del contorno general de la cabeza, característica que no se halla presente en la mayor parte de las especies de este género, asimismo ambas especies presentan una ornamentación estriada muy continua que es particularmente evidente en la cabeza, aunque las descripciones originales no dan mayor importancia a estos caracteres. Posteriores revisiones de material del Museo de Senckenberg muestran que estas características son bastante constantes en este grupo de especies y que la ornamentación se halla presente también en el pronoto y las patas, siendo particularmente importante la presencia de esta característica en las patas.

Estos hallazgos aunados a una filogenia preliminar que incluye también formas del Viejo Mundo indican que el grupo cesari es de fácil caracterización y constituye un grupo monofilético dentro de Elaphrothrips, como se expone más adelante. Sin embargo, uno de los inconvenientes más significativos en la clasificación de las especies de Elaphrothrips es que es más sencillo caracterizar los grupos de especies que el género mismo, lo cual constituye un enorme problema para la clasificación.

Kim \& Ludwig (1978) enfrentaron una situación similar al efectuar la revisión de Anoplura en su clasificación supragenérica, este problema fue particularmente evidente al tratar de definir la familia Hoplopleuridae, en la cual se definían con más claridad las subfamilias que la familia como tal. La solución práctica desde el punto de vista de la clasificación, fue elevar el rango de familias a estas subfamilias con lo que los grupos quedaban claramente definidos. La revisión de los grandes géneros dentro de Thysanoptera posiblemente culmine demostrando que estos géneros deben de segregarse en unidades más pequeñas y consistentes que ayuden en realidad a la clasificación del grupo.

Descripción de especies nuevas de Elaphrothrips. La especies descritas en este apartado pertenecen al grupo cesari y comparten el modelo general de los caracteres que lo definen.

\section{Elaphrothrips bussingi sp. nov.}

(Figuras 1A-B, 2C)

Diagnosis. Sedas posteroangulares y notopleurales agudas. Sedas del trocánter I agudas. Pelta reticulación homogénea.

Descripción. Cuerpo de color castaño claro. Sedas mayores de color castaño oscuro. Antenómeros I-II castaño claro, III amarillo con el 1/5 distal castaño, IV-V amarillo en la mitad basal, VI-VIII castaño claro. Alas hialinas. Cabeza sin seda posocular. Sedas posteroangulares y notopleurales de punta aguda (Fig. 1A). Sedas del trocánter I de punta aguda (Fig. 1B). Terguitos abdominales con dos pares de sedas retentorias y cuatro pares de sedas retentorias accesorias (Fig. 2C). Cuerpo fuertemente reticulado Pelta reticulada de forma homogénea. Esternitos estriados.

Medidas del holotipo (en $\boldsymbol{\mu m}$ ). Longitud total del cuerpo: 6700 , longitud de la cabeza: 750 , ancho de la cabeza detrás de los ojos: 275, longitud del proceso anterior del vértex: 100 y ancho: 160. Longitud del segmento $\mathrm{X}$ del abdomen (tubo): 625.

Medidas del macho (en $\mu \mathrm{m}$ ). Longitud total del cuerpo: 6700, longitud total del segmento X del abdomen (tubo): 625.

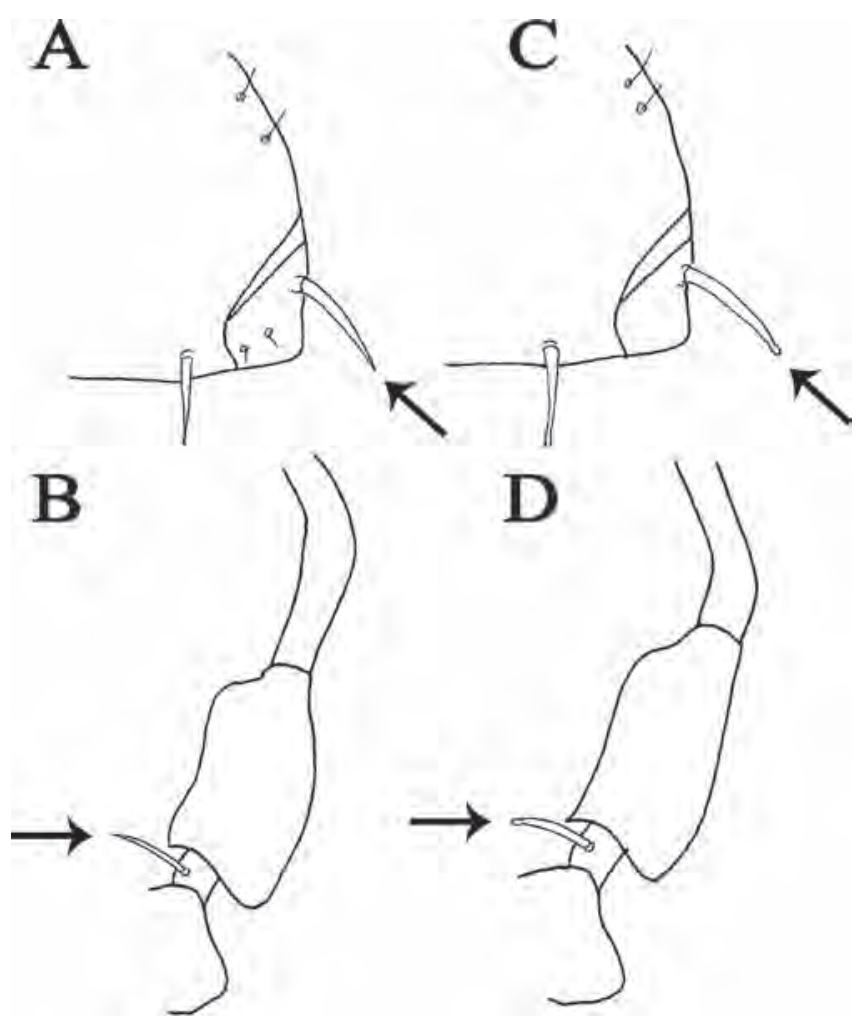

Figura 1. A-D. Elaphrothrips. A. Sedas posteroangulares y notopleurales agudas, B. Trocánter I con sedas agudas, C. Sedas posteroangulares y notopleurales redondeadas y D. Trocánter I con sedas redondeadas. A y B corresponden a E. bussingi; C y D a E. gomezlauritoi. 

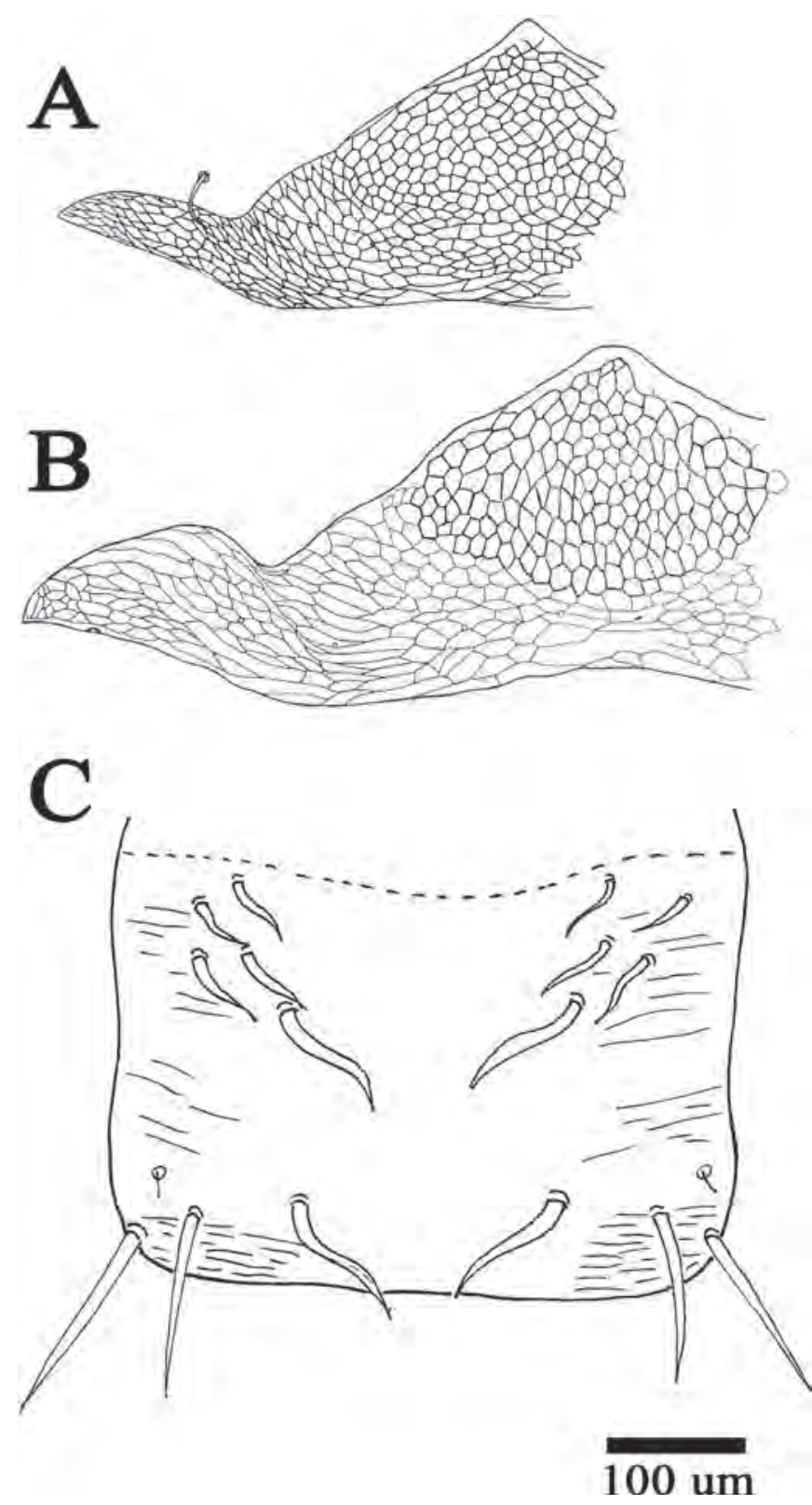

Figura 2. A-C. Elaphrothrips. A. Pelta de reticulación homogénea de E. gomezlauritoi, B. Pelta de reticulación heterogénea de E. fournieri y C. Detalle de sedas retentorias accesorias, segmento abdominal IV (figuras A y B representan la diferencia entre las dos peltas, tomadas de Johansen 1982).

Material examinado. Holotipo $q$ macróptera. COSTA RICA: Alajuela, Cachagua de Peñas Blancas. 21.VIII.1974 en tronco, (col. E. Klein/SMF). Paratipos:

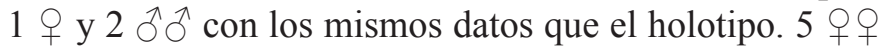
recolectadas en COSTA RICA: Alajuela, San Rafael de Palmares. 12.IX.1974 en tronco, (col. E. Klein/SMF). 2 우 recolectadas en COSTA RICA: Alajuela, San Isidro de Peñas Blancas. 9.X.1974.

Comentarios. Tanto la especie cesari como lorraineae presentan sedas del pronoto de punta aguda como en el caso de la especie nueva bussingi, pero se diferencia de la primera la coloración de los antenómeros III-VI amarilla con el borde distal castaño. Además, la reticulación de la pelta de cesari es heterogénea y el largo del cuerpo es mayor $(9837 \mu \mathrm{m})$. Tanto cesari como lorraineae presentan múltiples sedas accesorias de las sedas retentorias de las alas, mientras que esta especie nueva solo tiene cuatro pares de sedas accesorias.

Etimología. Esta especie está dedicada al científico e investigador William Bussing Burhaus por su contribución al conocimiento de la biología y la biodiversidad de peces del Neotrópico y por sus cualidades humanas.

\section{Elaphrothrips gomezlauritoi sp. nov.}

(Figuras 1C-D, 2A, 2C)

Diagnosis. Sedas posteroangulares y notopleurales de punta redondeada. Sedas del trocánter I de punta redondeada. Pelta reticulación homogénea.

Descripción. Coloración del cuerpo castaño claro. Antenómeros con patrón de coloración como sigue I-II castaño claro, III amarillo con el borde distal castaño, IV-V amarillo en la mitad basal, VI-VIII castaño claro. Sedas mayores de color castaño oscuro. Alas hialinas. Cabeza sin seda posocular. Sedas posteroangulares y notopleurales de punta redondeada (Fig. 1C). Sedas del trocánter I de punta redondeada y blanca (Fig. 1D). Cuerpo fuertemente reticulado. Esternitos estriados. Pelta con reticulación homogénea (Fig. 2A). Terguitos con dos pares de sedas retentorias y cuatro pares de sedas retentorias accesorias (Fig. 2C).

Medidas del holotipo (en $\mu \mathrm{m}$ ). Longitud total de cuerpo: 5125, longitud de la cabeza: 550, ancho de la cabeza detrás de los ojos: 225, longitud del proceso anterior del vértex: 70 y ancho: 130. Longitud del segmento $\mathrm{X}$ del abdomen (tubo): 425.

Medidas del macho (en $\mu \mathrm{m}$ ). Longitud total del cuerpo: 5075, longitud total del segmento $\mathrm{X}$ del abdomen (tubo): 375.

Material examinado. Holotipo $q$ macróptera. COSTA RICA: Alajuela, San Isidro de Peñas Blancas. 9.X.1974

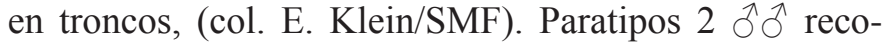
lectados en COSTA RICA: Alajuela, Cachagua de Peñas Blancas. 21.viii.1974 en tronco de Persea americana, aguacate. (col. E. Klein/SMF).

Comentarios. Las sedas notopleurales de esta especie nueva son de punta redondeada o roma, carácter que comparte con lorraineae, la única especie del grupo cesari que tiene esta característica. Al igual que en bussingi, lleva cuatro pares de sedas accesorias de las retentorias, mientras que en lorraineae son múltiples sedas accesorias de las sedas retentorias. 
Etimología. Esta especie está dedicada al científico e investigador Jorge Gómez Laurito por su contribución al conocimiento de la flora de Costa Rica y la región.

\section{Elaphrothrips fournieri sp. nov.}

(Figura 2B, 2C)

Diagnosis. Sedas posteroangulares y notopleurales de punta redondeada. Sedas del trocánter I de punta redondeada. Pelta reticulación heterogénea.

Descripción. Coloración del cuerpo castaño claro. Antenómeros con patrón de coloración como sigue I-II castaño claro, III amarillo con el borde distal castaño, IV-V amarillo en la mitad basal, VI-VIII castaño claro. Sedas mayores de color castaño oscuro. Alas hialinas. Cabeza sin seda posocular. Sedas posteroangulares y notopleurales de punta redondeada. Sedas del trocánter I de punta redondeada y blanca. Cuerpo fuertemente reticulado. Esternitos estriados. Pelta con reticulación heterogénea (Fig. 2B). Terguitos con dos pares de sedas retentorias y cuatro pares de sedas retentorias accesorias (Fig. 2C).

Medidas del holotipo (en $\boldsymbol{\mu m}$ ). Longitud total del cuerpo: 6125 , longitud de la cabeza: 750 , ancho de la cabeza detrás de los ojos: 275, longitud del proceso anterior del vértex: $100 \mathrm{y}$ ancho: 150. Longitud del segmento $\mathrm{X}$ del abdomen (tubo): 500 .

Hembra. Desconocida.

Material examinado. Holotipo đ macróptero. COSTA RICA: Alajuela, San Isidro de Palmares. 12.ix.1974 en tronco, (col. E. Klein/SMF). Paratipos $2 \widehat{\jmath} \widehat{\jmath}$ macrópteros con los mismos datos que el holotipo.

Comentarios. La única especie dentro del grupo cesari que presenta la pelta de reticulación heterogénea es $c e$ sari, la especie nueva fournieri tiene este carácter pero a diferencia de cesari, lleva las sedas notopleurales y posteroangulares de puntas redondeadas (romas) mientras que en cesari son de ápices agudos. Además, los antenómeros de cesari son de color amarillo del III-VI con la sección apical de color castaño, en la especie fournieri se presenta este patrón solo en los segmentos IV-V.

Etimología. Esta especie está dedicada al científico e investigador Luis Fournier Origi por su aporte al conocimiento de los procesos ecológicos de las plantas en la región del Neotrópico.

\section{DISCUSIÓN}

\section{Definición de Elaphrothrips Elaphrothrips Buffa, 1909}

Mound \& Palmer (1983) presentan una revisión de la subfamilia Idolothripinae y proponen una clasificación supragenérica diferente a la establecida hasta ese momen- to. Jacot-Guillarmod (1978) considera que la clasificación supragenérica dentro de Idolothripinae debe considerar los siguientes grupos:

Idolothripini

Idolothripina: 32 géneros Compsothripini

Atractothripina: 1 género Compsothripina: 3 géneros

Hystricothripina; 3 géneros Hartwigia: 1 género

Megathripina: 2 géneros Emprosthiothripini

Apelaunothripina: 2 géneros Emprosthiothrips

Cryptothripini Pygidiothripini

Cryptothripina; 22 géneros Pygidiothrips

Allothripina: 6 géneros Pygothripini

Gastrothripina: 13 géneros Pygothrips

Diceratothripina: 18 géneros

Mound \& Palmer (1983) proponen la siguiente clasificación supragenérica

Pygothripini

Pygothripina

Macrothripina

Allothripina

Idolothripini

Compsothripina

Elaphrothripina

Gatrothripina

Idolothripina

Hystricothripina

Diceratothripina

La clasificación propuesta por Mound \& Palmer (1983) se fundamenta en los resultados de una matriz de datos de ocho caracteres para analizar las relaciones de nueve taxa presentado en un diagrama. El uso de esta matriz en un programa de evaluación y búsqueda de la reconstrucción filogenética más precisa y de mayor parsimonia, nos indica que esta matriz cuenta tan solo con cinco caracteres informativos, tres de los caracteres resultan inútiles para la definición de los grupos. Esto significa que las relaciones se hallan fundamentadas en tan solo cinco caracteres para resolver nueve taxa, lo cual es insuficiente. Lamentablemente, los autores no indicaron cual grupo se utilizó como grupo externo de comparación, ni establecen qué programa y supuestos se utilizaron al momento de efectuar la reconstrucción filogenética. Por este motivo, se ha considerado para la reconstrucción filogenética el agregar una hilera Ho que representa un supuesto ancestro hipotético.

Los caracteres considerados por Mound \& Palmer (1983) fueron:

1) Estiletes maxilares largos 0 cortos 1

2) Segmento antenal IV con 2 conos sensoriales 0, 4 conos sensoriales 1,3 conos sensoriales 2 
3) Suturas esternopleurales metatorácicas presentes 0 , ausentes 1

4) Sedas retentorias de los terguitos 1 par 0, 2 pares 1

5) Tubo glabro 0 , pubescente 1

6) Placas del praepectum presentes 0 , ausentes 1

7) Conos sensoriales de la antena delgados 0 , fuertes 1

8) Sensor del palpo maxilar pequeño 0 , grande 1

Al efectuar el análisis se ha utilizado la estrategia exhaustiva, la cual ha dado 18 árboles igualmente probables. El consenso estricto muestra que el único grupo que se sostiene como grupo natural es el clado (Macrothripina (Elaphrothripina (Idolothripina, Hystricothripina))), los demás grupos forman una politomía basal. La propuesta de parentescos de Mound \& Palmer (1983) no coincide con ninguna de las 18 topologías obtenidas. La longitud mínima obtenida es de nueve pasos, en tanto que en la figura 1 presentada por estos autores hay una longitud de 39 pasos, lo que la hace una posibilidad menos aceptable.

La clasificación de Mound \& Palmer (1983) que coincide con el análisis efectuado en el grupo de los Idolothripini, no incluye a Macrothripina, la cual se halla contenida en Pygothripini, por lo que esta clasificación es parafilética y en consecuencia artificial y debe ser corregida en pos de una clasificación natural de esta subfamilia. Del análisis formal de la matriz de datos proporcionada por Mound \& Palmer (1983) se desprende que el dendrograma presentado por estos autores en la figura 1 no obedece a los resultados más probables de los parentescos y lo que ilustra dicho dendrograma. Por otra parte, de los caracteres informativos comprendidos del 1 al 5 (ver lista de caracteres arriba), los únicos que presentan una inercia filogenética alta (ri $>0,8)$ son los caracteres 2 a 5 (Diniz-Filho 2000, Retana-Salazar \& Retana-Salazar 2008). Esto indica que el carácter referido al número de sedas retentorias es uno de los de mayor valor para determinar parentescos filogenéticos, y en consecuencia para indicar una buena estructuración filogenética, tal y como Mound \& Palmer (1983) lo consideran en sus claves de identificación.

Posteriores trabajos han demostrado que las inferencias de clasificación supragenéricas son incorrectas y en gran medida estos errores derivan de la alta homoplasia de este grupo de insectos (Gauld \& Mound 1982). Por lo tanto, la inferencia en ausencia de análisis filogenéticos formales fue necesaria mientras no existió la tecnología necesaria para efectuar mejores investigaciones que nos permitan obtener hipótesis de clasificación más reales. Retana-Salazar (2009) después de varios análisis filogenéticos demostró que los grupos cercanos a Zeugmatothrips conforman un grupo parafilético entre las especies de este género y otros géneros descritos como Cyphothrips (Retana-Salazar \& Soto-Rodríguez 2001). Un nuevo análisis más detallado pone a prueba la hipótesis de Mound \&
Palmer (1983) según la cual las similitudes morfológicas entre algunos Hystricothripina y algunos grupos dentro de Elaphrothripina se deben a convergencias. Estas evidencias fueron derivadas de la observación pero no de un análisis formal de una matriz de caracteres. El análisis filogenético de los grupos con similitudes indican que las convergencias interpretadas por Mound \& Palmer (1983) no son homoplasias sino más bien caracteres homólogos que justifican este grupo natural, por lo que Retana-Salazar (2009) considera la siguiente clasificación supragenérica:

Pygothripini
Pygothripina
Allothripina
Macrothripina
Idolothripini
Elaphrothripina
Idolothripina

Compsothripina Gastrothripina Diceratothripina Hystricothripina Anactinothripini Anactinothripina Zeugmatothripina

\section{Problemas en el criterio de especie dentro de Elaphrothrips}

Mound \& Palmer (1983) consideran siete nombres genéricos como sinónimos de Elaphrothrips y consideran 150 especies en el mismo, de las cuales 37 fueron considerados sinonimias. Mound \& Marullo (1996) indican que los resultados de Mound \& Palmer (1983) se fundamentaron en largas series estudiadas de especímenes de Asia y África, en las cuales reconocieron patrones de alometría particularmente en los machos. Sin embargo, esta variación alométrica puede ser engañosa. Como ha sido establecido en múltiples artículos, la alometría no es una percepción del observador a través del examen de largas series, sino la determinación de una proporción orgánica en el desarrollo corporal de los individuos (Gould 1966). Numerosas revisiones históricas muestran esta necesidad de formulación matemática para determinar la existencia de alometría (Gayson 2000). La alometría ligada al sexo ha sido ampliamente estudiada desde 1920 (Champy 1924, 1929, Gayson 2000) y para ello es necesario el examen estadístico de largas series de individuos.

Los estudios que involucran análisis serios de datos tratados estadísticamente no son habituales en Thysanoptera. A pesar de su valor como herramienta en la determinación de caracteres de utilidad en la segregación de especies (Kobro \& Rafoss 2006), no es utilizada con frecuencia por los taxónomos alfa y como es evidente no es suficiente con examinar las series de especímenes para determinar la existencia de alometría y mucho menos 
para poder determinar el valor de estos caracteres en la segregación de especies.

Mound \& Marullo (1996) señalan que el concepto de especie utilizado por Johansen $(1983,1986)$ con respecto a Elaphrothrips es más estrecho que el considerado por él, y esto redunda en una considerable cantidad de formas tratadas como especies. Estos autores critican que las claves de Johansen son excesivamente complejas. Sin embargo, ha habido críticas que indican que el trabajo de Mound \& Marullo (1996) incurre en una serie de errores de fondo y forma, entre ellos algunas iconografías no apropiadas que no permiten claridad en la identificación de las especies, como algunas omisiones relacionadas con caracteres importantes en las claves que las hacen de difícil acceso (Bhatti 1998). Con lo cual no se soluciona el problema de las claves y la identificación de las especies.

El criterio de especies con amplia variabilidad morfológica debe ser estudiado con cautela, y sobre todo, debe ser demostrado a través del estudio de largas series en las cuales se pueda establecer de forma estadística los ámbitos de variación de los caracteres en las diferentes especies (Kobro \& Rafoss 2006) o del estudio de la biología de la especie (Retana-Salazar \& Soto-Rodríguez 2002). Por otra parte, numerosos ecólogos han demostrado que las especies abundantes son las menos y que la diversidad aumenta hacia las regiones neotropicales (Guttman 1999). Una alta diversidad a menudo se refleja en numerosas especies con bajas poblaciones (Krebs 1985, Retana-Salazar 2009), lo cual hace difícil en las regiones neotropicales la obtención de largas series de individuos que permitan estudios de tipo estadístico morfométrico. Esta fue, posiblemente una de las razones del por qué Mound \& Palmer (1983) solo analizaron largas series de individuos de unas pocas especies de Asia y África.

Los taxónomos del Nuevo Mundo deben enfrentar problemas muy diferentes, desde el punto de vista ecológico, a los que presentan los taxónomos del Viejo Mundo. Por ello, se debe considerar con cierta cautela la extrapolación de modelos de variación morfológica derivados del estudio exhaustivo de especies del Viejo Mundo, acerca de la respuesta a ambientes muy homogéneos como explicación a los fenómenos registrados en áreas de alta variación ambiental. De esta forma, en la última década una serie de modelos matemáticos han demostrado que la especiación ecológica es posible en simpatría y lo demuestran diversos ejemplos, entre los cuales se incluyen los de insectos fitófagos adaptados a diferentes hospederos, peces adaptados a diferentes hábitats en los lagos, aves adaptadas a diferentes fuentes de alimento y plantas adaptadas a diferentes tipos de suelo o polinizadores (Hendry 2009).

Estas evidencias podrían indicar que sitios de alta diversidad como las regiones neotropicales, y con una alta cantidad de microhábitats podrían ser enormes fuentes de especiación promovida por factores ecológicos. Desde esta perspectiva y en ausencia de una verdadera demostración de la existencia de variaciones alométricas en este grupo de insectos, debe tomarse en cuenta la posición de Johansen $(1983,1986)$ al considerar un concepto de especie más estrecho, como una opción que refleja con mayor exactitud la situación biológica de las especies de thrips en el neotrópico.

Entre los taxónomos alfa es común que se esgrima el argumento de hallar en forma esporádica algunos individuos con caracteres de dos especies, con lo que algunos taxónomos asumen hibridación o variabilidad intraespecífica, y con este argumento se sinonimizan especies aunque no presenten evidencias de que en realidad se trate de las mismas especies (Goldarazena et al. 2008). Estas conclusiones ignoran el análisis de numerosos trabajos de investigación, que han demostrado que en grupos ampliamente aceptados como especies diferentes, a menudo se violenta este criterio. Las estimaciones actuales indican que al menos el $25 \%$ de las especies de plantas y el $10 \%$ de las especies de animales hibridizan exitosamente al menos con otra especie (Hendry 2009).

Por otra parte, estos taxónomos que a menudo son seguidores del concepto biológico de especie (CBE), consideran que una variación exhibida por poblaciones con una distribución disjunta, indica la presencia de una especie diferente. Sin embargo, es complicado utilizar la justificación para la especiación esgrimida por los seguidores del CBE, tomando en cuenta que en grupos de distribución alopátrica es muy difícil saber si habría colapso en caso de hallarse en contacto (Hendry 2009). Además el CBE no puede ser aplicado a muchos organismos como aquellos que presentan reproducción asexual o que se hallan extintos (Retana-Salazar \& Retana-Salazar 2004, 2008, Hendry 2009).

Estas razones entre otras, son las consideradas por algunos investigadores que promueven el uso de otros conceptos de especie, fundamentados en la magnitud de las diferencias morfológicas y genéticas entre grupos. El problema surge al tener que definir cuál debe ser la magnitud de las diferencias para considerar una separación de especies (Hendry 2009). Aunque algunos estiman que cualquier cambio asociado a la estructura filogenética del grupo sería suficiente para justificar la segregación (Retana-Salazar 2007). De igual forma, el análisis de la estructura del grupo en base a los caracteres del grupo permite definir los caracteres que determinan el linaje y cuales caracteres definen ramificaciones del linaje inicial. Sin embargo, ésto implica el conocimiento de la filogenia de los diversos grupos, lo cual suele ser difícil. 
De aquí que la definición de un posible grupo ancestro, ayude a definir el sentido de los cambios en ausencia de una topología determinada, y poder asumir los posibles grupos cadena que definen los linajes y las divergencias con respecto a éstos, y de esta forma definir la segregación de las especies (Retana-Salazar \& Retana-Salazar 2004, 2008).

\section{Los grupos de especies dentro de Elaphrothrips}

Como lo establecen Mound \& Palmer (1983), Elaphrothrips es el más diverso y de más amplia distribución de los géneros incluidos en Idolothripinae, se halla en todas las áreas tropicales y estos autores destacan que las especies de este género son sustituidas por las de Mecynothrips en el Este de Sulawesi. Mound \& Palmer (1983) conjeturan que en su criterio los grupos de especies hallados en América del Sur, África e India no presentan diferencias sustanciales. Sin embargo, es necesario tomar en cuenta que uno de los factores que más dificulta el estudio de este grupo de especies es la alta plasticidad de sus caracteres (Retana-Salazar \& Soto-Rodríguez 2002).

Johansen (1982) presenta la revisión más exhaustiva hasta el momento escrita de este género, incluyendo claves ilustradas de los grupos de especies y de las especies incluidas en los mismos. Uno de los mayores problemas se presenta al organizar la información de forma clara cuando se trata de grupos de especies tan diversas, en los cuales incluso la definición de los grandes grupos se vuelve compleja. Por su parte algunos autores han criticado la excesiva dificultad en el manejo de las claves de este trabajo, considerando que algunos de los caracteres utilizados no disciernen con claridad entre los grupos de especies (Mound \& Marullo 1996). Este mismo problema se detecta en la clave a nivel de género presentada por Mound \& Palmer (1983), donde la segregación final del género se efectúa utilizando caracteres complejos de los machos, los cuales no siempre se hallan disponibles.

Johansen (1986) presenta una nueva revisión de algunos de los grupos de especies, enmendando algunos supuestos errores de la clasificación taxonómica propuesta por él mismo. Incluye en este caso una clave de las especies, que es un excelente intento por ofrecer una herramienta de identificación y presenta el primer análisis de filogenia del grupo. La clave para especies de Costa Rica presentada por Mound \& Marullo (1996) se halla fundamentada en los caracteres del macho, lo que complica en mucho su uso, además de que la misma separa y reconoce tan solo siete especies, siendo una herramienta de poca utilidad para aquellos que no se hallan estrechamente familiarizados con el grupo. Por su parte, Retana-Salazar \& Soto-Rodríguez (2002) presentan una clave más amplia y que utiliza otros caracteres morfológicos para las especies de Costa Rica y en la misma se incluyen 16 especies, de las cuales tres son nuevas.

\section{Variación morfológica en Elaphrothrips}

Mound \& Palmer (1983) consideran 37 sinonimias de las 150 especies registradas en este género en ese momento. Los autores señalan que esta decisión fue tomada después de la observación de largas series de especímenes de Asia y África y después del reconocimiento de complejos patrones alométricos, particularmente en los machos (Palmer \& Mound 1978). Sin embargo, en ninguna de estas publicaciones se presentan datos de series estadísticamente significativas, que permitan un análisis morfométrico real en el cual las pruebas estadísticas determinen la significancia de los caracteres y su asociación con la variación de tamaño. Esto nos indica que la llamada alometría, que es una condición que solo puede ser comprobada mediante el uso de modelos matemáticos y comparaciones estadísticas (García-Barros 1999), ha sido tan solo una apreciación subjetiva de estos autores.

Este tipo de aseveraciones por parte de los taxónomos con frecuencia inducen a errores en la consideración de patrones morfológicos que sirvan para la segregación de especies o grupos de especies. La observación de al menos 10 ejemplares de Elaphrothrips propinquus de la colección del Senckenberg Museum indica que esta especie puede ser un complejo de especies. En estos especímenes se puede observar que, aunque son muy similares entre sí, los de mayor tamaño presentan como es común en el grupo dos pares de sedas retentorias en los terguitos abdominales, pero los más pequeños presentan solo un par.

Aplicando los postulados de Mound \& Palmer (1983) y Mound \& Marullo (1996) deberíamos considerar que estas variaciones son debidas a la variación del tamaño en los especímenes estudiados. Sin embargo, Mound \& Palmer (1983) consideran que la presencia de uno o dos pares de sedas retentorias en los terguitos abdominales es una de las características importantes para la separación de tribus dentro de Idolothripinae y señalan que entre las pocas excepciones se halla una única especie de Elaphrothrips que solo presenta un par de sedas retentorias. Es obvio que si un carácter es considerado de importancia en la separación de grupos supragenéricos, este carácter no puede ser de fácil modificación. En consecuencia sería obvio pensar que los especímenes de Elaphrothrips surinamensis que presentan un solo par de sedas retentorias deberían considerarse como otra especie.

El argumento de mayor peso esgrimido por los taxónomos para sustentar esta conjetura se basa en los datos 
de recolección, asumiendo que los organismos recolectados en un mismo momento y lugar son la misma especie. Dentro de Thysanoptera esta forma de razonamiento es peligrosa, ya que son innumerables los casos en que se pueden recolectar diferentes especies en conjunto. Por ejemplo, en invernaderos de Costa Rica se han recolectado las especies Frankliniella occidentalis, F. insularis, $F$. panamensis y $F$. cephalica en trampas pegajosas de un mismo invernadero en camas de la misma especie de planta. En plantas contiguas de Senecio se han recolectado especímenes de F. bagnalliana, F. fallaciosa y F. minuta. Mound \& Marullo (1996) describen una especie nueva Frankliniella lorena, a partir de un único espécimen recolectado junto con una serie de ejemplares de F. fulvipennis. Dentro del género Ozothrips descrito por Mound \& Palmer (1983), estos autores reconocen inicialmente tres especies, en una posterior revisión del material incluido en $O$. eurytis indican que seis especímenes considerados similares a esta especie fueron luego incluidos en el material tipo de O. tubulatus (Mound \& Walker 1986). Estos ejemplos indican que la distribución simpátrica es común en Thysanoptera y no es posible utilizar este argumento para justificar la variabilidad dentro de los organismos obtenidos en una única recolecta, y ni tan siquiera es útil cuando se trata de un mismo hospedero.

Esto mismo sucede al analizar la fauna dentro de las agallas de Gynaikothrips uzeli y Gynaikothrips garitacambroneroi donde se hallan junto con cualquiera de estas especies a Androthrips ramachandrai (Garita-Cambronero \& Lizano-Fallas 2006, Held \& Boyd 2008). Además, una de las especies más conflictivas ha sido G. garitacambroneroi, la cual se diferencia de $G$. uzeli por la genitalia del macho, pero tanto G. uzeli como G. garitacambroneroi se encuentran en agallas de Ficus benjamina. Goldarazena et al. (2008) han propuesto que esta especie es sinónimo de G. uzeli, pero sin una correcta revisión del material tipo de la especie G. garitacambroneroi. Estudios de la ultraestructrura de la agalla de esta especie indican que ésta presenta un desarrollo diferente al de las agallas producidas por otros thrips en el mismo hospedero (RetanaSalazar \& Sánchez-Chacón 2009). El trabajo en el que se describe por primera vez la especie G. garitacambroneroi presenta un análisis comparativo de las variaciones morfológicas dentro de las especies del grupo uzeli y las variaciones dentro de los especímenes recolectados en diferentes localidades de Mesoamérica y el Caribe, de donde se desprende que los caracteres utilizados convencionalmente en la separación de estas especies no son confiables (Retana-Salazar 2006). Estudios ultraestructurales de los thrips hallados en agallas en Ficus benjamina en Costa Rica y México indican que dentro de estas agallas se pueden diferenciar con claridad tres segregados morfo- lógicos sin presencia de formas intermedias (RodríguezArrieta \& Retana-Salazar 2010). Estos datos se ajustan a las características necesarias para establecer que existen especies simpátricas (Mayr 1969).

Las evidencias indican que dentro de los grupos de Thysanoptera deben ser más comunes de lo que se piensa las especies crípticas y los patrones de especiación simpátricos. Las especies son eventos biológicos donde la morfología es un excelente indicador que siempre debemos buscar y establecer, pero no porque las variaciones sean muy pequeñas indica que las mismas deben de ser atribuidas a variación morfológica de alguna especie conocida. Son los patrones biológicos asociados a patrones morfológicos los que pueden dar la clave acerca de la diferenciación de las especies en estos casos y muchas veces pequeñas diferencias morfológicas constantes en especies cuyas poblaciones son simpátricas son los mejores indicadores de especies diferentes (Mayr 1969).

\section{MATERIAL REVISADO}

Elaphrothrips affinis Bagnall 1908. Material de Costa Rica. Localidad tipo: Nicaragua

Elaphrothrips angustatus Bagnall 1910. Material de Costa Rica. Localidad tipo: Venezuela

Elaphrothrips bilineatus Priesner 1933. SMF T 9728 Tipo macho. Localidad tipo: México

Elaphrothrips cesari Retana-Salazar \& Soto-Rodríguez 2002. CIEMIC Holotipo macho. Localidad tipo: Costa Rica

Elaphrothrips championi Bagnall 1910. Material de Costa Rica. Localidad tipo: Guatemala

Elaphrothrips dampfi Hood 1940. CIEMIC material de Costa Rica. Localidad tipo: México

Elaphrothrips foveicollis Bagnall 1908. Material de Costa Rica. Localidad tipo: Guatemala

Elaphrothrips grandis Bagnall 1910. Material de Costa Rica. Localidad tipo: Guatemala

Elaphrothrips jacotguillarmodi Johansen 1979. Material de Costa Rica. Localidad tipo: Perú

Elaphrothrips longiceps Bagnall 1908. Material de Costa Rica. Localidad tipo: México

Elaphrothrips lorraineae Retana-Salazar \& Soto-Rodríguez 2002. CIEMIC Holotipo macho. Localidad tipo: Costa Rica

Elaphrothrips magnus Johansen 1979. Material de Costa Rica. Localidad tipo: Perú

Elaphrothrips neoleonensis Johansen 1979. Material de Costa Rica. Localidad tipo: México

Elaphrothrips priesneri Bagnall 1926 (Dicaiothrips breviceps Priesner 1921). SMF T 7470 hembra. Localidad tipo: Paraguay 
Elaphrothrips propinquus Bagnall 1910. Material de Costa Rica. Localidad tipo: Venezuela

Elaphrothrips quirosi Johansen \& Retana-Salazar 1999. CIEMIC holotipo. Localidad tipo: Costa Rica

Elaphrothrips surinamensis Priesner 1925. Material de Costa Rica. Localidad tipo: Surinam

Elaphrothrips tener Priesner 1925. SMF T 12432 Tipo macho. Localidad tipo: México

Elaphrothrips valerioi Retana-Salazar \& Soto-Rodríguez 2002. CIEMIC Holotipo hembra, Paratipo hembra. Localidad tipo Costa Rica

\section{ESPECIES DE MESOAMÉRICA}

albospinosus Moulton 1929 México angusticeps Crawford 1910 México aztecus Hood 1941 México cognatodampfi Johansen 1980 México cognatograndis Johansen 1978 México garcialdretei Johansen 1980 México herculeus Johansen 1980 México insperatus Johansen 1979 México lewisi Johansen 1980 México maya Johansen 1986 México morelensis Johansen 1980 México neodampfi Johansen 1979 México neolongiceps Johansen 1979 México oaxacaensis Johansen 1980 México occidentalis Johansen 1980 México paradampfi Johansen 1979 México propeherculeus Johansen 1980 México pseudotuberculatus Johansen 1980 México schultzei Priesner 1933 México tepoztlanensis Johansen 1986 México villai Johansen 1980 México zacualtipanensis Johansen 1983 México

AGRADECIMIENTOS. Al Dr. Richard zur Strassen del Senckenberg Museum, Frankfurt (SMF), Alemania por el préstamo del material tipo de Elaphrothrips revisado y descrito en este estudio.

\section{LITERATURA CITADA}

Bhatti, J. S. 1998. The Thrips of Central and South America by Laurence A. Mound and Rita Marullo. A Review. Journal of Pure and Applied Zoology, 5: 141-146.

Champy, C. 1924. Sexualite et hormones. Doin, Paris. 150 pp.

Champy, C. 1929. La croissance dysharmonique des caractères sexuels accessoires. Nature Zoologie, 193-244.

Diniz Filho, J. A. 2000. Métodos Filogenéticos Comparativos. Editorial Holos. Brasil, 120 pp.

García-Barros, E. 1999. Implicaciones ecológicas y evolutivas del tamaño en los artrópodos. Boletín de la Sociedad Entomológica Aragonesa 26: 657-678.
Garita-Cambronero, J. \& Lizano-Fallas, V. 2006. Determinación de dimorfismo sexual de Gynaikothrips garitacambroneroi (Thysanoptera: Phlaeothripidae) inductor de agallas en Ficus benjamina. Métodos en Ecología y Sistemática, 1: 10-14.

Gauld, I. D. \& Mound, L. A. 1982. Homoplasy and the delineation of holophyletic genera in some insect groups. Systematic Entomology, 7: 73-86.

Gayson J. 2000. History of the concept of allometry. American Zoologist, 40: 748-758.

Goldarazena, A., Mound, L.A. \& zur Strassen, R. 2008. Nomenclatural problems among Thysanoptera (Insecta) of Costa Rica. Revista de Biología Tropical, 56: 961-968.

Gould, S. J. 1966. Allometry and size in Ontogeny and Phylogeny. Biological Reviews, 41: 587-638.

Guttman, B. S. 1999. Biology. The Structure of Biological Communities. WCB McGraw-Hill.

Held, D. \& Boyd, J. R. 2008. New records of Gynaikothrips uzeli (Zimmerman) (Thysanoptera: Phlaeothripidae) on Ficus benjamina in Texas and O'ahu, Hawaii, USA. Pan-Pacific Entomology, 84: 77-80.

Hendry, A. P. 2009. Speciation. Nature, 458: 162-164.

Jacot-Guillarmod, C. F. 1978. Catalogue of the Thysanoptera of the World, part. 5. Annual Cape Province Museum (Natural History), 7: 1257-1556.

Johansen, R. M. 1982. El género Elaphrothrips Buffa, 1909 (Thysanoptera: Phlaeothripidae) en el Continente Americano; su Sistemática, Evolución, Biogeografía, Ecología y Biología. Monografías. Instituto de Biología Universidad Nacional Autónoma de México, No. 1: 1-267.

Johansen, R. M. 1983. Nuevos thrips tubulíferos (Insecta: Thysanoptera) de México. XI. Anales del Instituto de Biología Universidad Nacional Autónoma de México, Serie Zoología, 53: 55-89.

Johansen, R. M. 1986. Nuevos conceptos taxonómicos y filogenéticos del género Elaphrothrips Buffa, 1909 (Thysanoptera: Phlaeothripidae), del continente Americano y descripción de dos especies nuevas. Anales del Instituto de Biología Universidad Nacional Autónoma de México, Serie Zoología, 3: 745-868.

Kim, K. C. \& Ludwig, H. W. 1978. The family classification of the Anoplura. Systematic Entomology, 3: 249-284.

Kobro, S. \& Rafoss, T. 2006. Identification of adult males and females of Hoplothrips species (Thysanoptera: Tubulifera) known form Norway, and some deductions on their life history. Entomologica Fennica, 17: 184-192.

Krebs, C. J. 1985. Ecology: The Experimental Analysis of Distribution and Abundance. Third Edition. Harper and Row, New York, 800 pp.

Mayr, E. 1969. Principles of systematic zoology. McGraw-Hill, New York, 416 pp.

Mound, L. A. 1968. A review of R.S. Bagnall's Thysanoptera collections. Bulletin of the British Museum (Natural History. Entomology, 11: 1-181.

Mound, L. A. \& Marullo, R. 1996. The thrips of Central and South America: An Introduction (Insecta: Thysanoptera). Memoirs on Entomology. 488 pp.

Mound, L. A. \& Palmer, J. M. 1983. The generic and tribal classification of spore-feeding Thysanoptera (Phlaeothripidae: Idolothripinae). Bulletin of the British Museum (Natural History). Entomology, 46: 1-174.

Mound, L. A. \& Walker, A. K. 1986. Tubulifera (Insecta: Thysanoptera). Fauna of New Zealand, 10: 1-140. 
Palmer, J. M. \& Mound, L. A. 1978. Nine genera of fungus-feeding Phlaeothripidae (Thysanoptera) from the Oriental Region. Bulletin of the British Museum (Natural History). Entomology, 37: 153-215.

Retana-Salazar, A. P. 2006. Variación morfológica en el complejo Gynaikothrips uzeli-ficorum. Métodos en Ecología y Sistemática, 1: 1-9.

Retana-Salazar, A. P. 2007. El grupo genérico Hoodothrips (Terebrantia: Heliothripidae). Acta Zoológica Lilloana, 51: 15-38.

Retana-Salazar, A. P. 2009. Monografía de los grupos genéricos Anactinothrips-Zeugmatothrips (Tubulifera: Idolothripinae). Editorial del Instituto Centroamericano para la Investigación en Biología y Conservación (CIBRC), Costa Rica, 140 pp.

Retana-Salazar, A. P. \& Soto-Rodríguez, G. A. 2001. Filogenia del género neotropical Zeugmatothrips (Idolothripinae: Hystrichothripina). Revista Gayana, 65: 119-128.

Retana-Salazar, A. P. \& Soto-Rodríguez, G.A. 2002. Especies de Elaphrothrips (Thysanoptera: Phlaeothripidae: Idolothripinae) ha- lladas en Costa Rica, con la descripción de tres especies nuevas. Acta Zoológica Mexicana (n. s.), 85: 129-138.

Retana-Salazar, A. P. \& Retana-Salazar, S.A. 2004. Hacia una lógica simple en la determinación de grupos biológicos: la especie y los grupos supraespecíficos. Revista de Biología Tropical, 52: 19-26.

Retana-Salazar, A. P. \& Retana-Salazar, S. A. 2008. Entropía Biológica: Especies y Filogenia. Editorial del Instituto Centroamericano para la Investigación en Biología y Conservación (CIBRC), Costa Rica, 167 pp.

Retana-Salazar, A. P. \& Sánchez-Chacón, E. 2009. Anatomía de la agalla en Ficus benjamina (Moraceae) asociada a "thrips" (Tubulifera: Phlaeothripidae). Revista de Biología Tropical, 57: 179-186.

Rodríguez-Arrieta, J. A. \& Retana-Salazar, A.P. 2010. Ultra-structure variability of Gynaikothrips uzeli-ficorum (Thysanoptera: Phlaeothripidae) complex in Ficus benjamina from Mexico and Costa Rica. Brenesia, 73-74: 89-97. 